\title{
Endogenous neighborhood formation in a local coordination model with negative network externalities
}

\author{
Giorgio Fagiolo*
}

\begin{abstract}
Laboratory of Economics and Management, Sant'Anna School of Advanced Studies, P.zza Martiri della Libertà, 33, I-56127 Pisa, Italy
\end{abstract}

\begin{abstract}
The paper studies the evolution of coordination in a local interaction model where agents can simultaneously choose the strategy to play in the game and the size of their neighborhood. We focus on pure-coordination games played by agents located on one-dimensional lattices and we assume that network externalities become eventually negative as neighborhood sizes increase. We show that the society almost always converges to a steady-state characterized by high levels of coordination and small neighborhood sizes. We find that neighborhood adjustment allows for higher coordination than if interaction structures were static and that large populations attain higher coordination provided that average initial neighborhood sizes are not too small.

(C) 2004 Elsevier B.V. All rights reserved.
\end{abstract}

JEL classification: $\mathrm{C} 72 ; \mathrm{C} 73$

Keywords: Learning; Coordination; Nearest-neighbor interactions; Endogenous neighborhood formation; Negative network externalities

\section{Introduction}

The outcome of a coordination game repeatedly played in large societies might be crucially affected by the coevolution between interaction structures and individual choices.

Traditionally, this issue has been investigated by assuming exogenously fixed networks. In these models, agents are supposed to repeatedly play a $2 \times 2$ coordination

\footnotetext{
* Tel.: +39-050 883 343; fax: +39-050 883344 .

E-mail address: fagiolo@sssup.it (G. Fagiolo).
} 
game either against any other player in the population (cf. Kandori et al., 1993; Young, 1996) or against a fixed, typically small, set of 'relevant others' (see Blume, 1993; Ellison, 1993; Young, 1998). The basic rationale is that, in many situations, the time-scale at which agents choose their opponents in the game is very long as compared to that at which they update their stage-game strategies. Consequently, it may be plausible to study the long-run properties of population learning as if interaction structures were static (cf. Skyrms and Pemantle, 2000).

However, the evolution of individual choices through time might also affect agents' expectations about future payoffs from interactions. A player might then change her idea on who is in fact 'relevant' and avoid further interactions (or, respectively, start interacting) with groups of other agents if she expects that this would lead to a decrease (or, respectively, an increase) in net payoffs. In such circumstances, the speed at which agents revise the set of their partners is (at least) comparable to the frequency at which they update their strategy. The details of the process governing the coevolution between strategies and interaction structures may therefore have non-trivial consequences on long-run coordination patterns (see Kirman, 1997).

Coordination games in presence of endogenous partner selection has been studied in the last few years in the context of dynamic network formation (see e.g. Goyal and Vega-Redondo, 2001; Jackson and Watts, 2000; Droste et al., 2000). The basic assumption shared by existing formalizations is that strategy and network updating are driven by 'positive network externalities'. In these models, as long as the unit cost of keeping open a connection with another player is not too large, agents always prefer more coordinated partners.

In this paper, we depart from this assumption by studying a setting characterized by network externalities which may be positive when agents hold small neighborhoods sizes, but become eventually negative when neighborhood sizes are very large. We argue that in some cases (e.g. network congestion effects), the positive contribution to individual payoffs from adding a new, coordinated, partner may be offset by negative effects induced by network sizes. To address this issue, we present a dynamic model of coordination in which agents can simultaneously choose the strategy to play in the game and select the agents with whom to play the game. We consider a population of myopic individuals located on a circle who repeatedly play a pure coordination game with their 'nearest neighbors'. We assume that holding neighbors is costly and that, from time to time, agents are allowed to slightly shrink or enlarge the 'radius' of their current neighborhood by maximizing expected net payoffs.

We show that the society almost always converge to a (non-unique) steady state in the long-run. We find that both full coordination and coexistence of conventions may be possible in a steady state. In turn, strategy configurations characterized by partial coordination may be sustained by both homogeneous and heterogeneous interaction structures. We then explore how system parameters (i.e. population size, unit link cost and probability of neighborhood revision) affect the likelihood of converging to steady states characterized, on average, by high (low) coordination and small (large) neighborhood sizes. We find that the system is able to robustly reach very high coordination levels, despite the presence of negative local network externalities. Moreover, we show that average coordination in presence of a non-zero (although small) 
frequency of neighborhood adjustment is higher than if interaction structures were static. Finally, we find that large societies coordinate better than small ones, provided that initial average neighborhood sizes are sufficiently high.

The rest of the paper is organized as follows. In Section 2, we review related works and we discuss the extent to which our model departs from existing literature. In Section 3, we formally describe the model. Section 4 deals with the characterization of absorbing states, while computer simulation results on the average long-run behavior of the system are reported in Section 5. Finally, Section 6 discusses extensions and future research.

\section{Coordination and endogenous neighborhood formation}

The question whether endogenous network formation may affect long-run coordination in populations of adaptive agents has been recently addressed in setups where network externalities are positive. In these formalizations (see e.g. Goyal and VegaRedondo, 2001), one considers agents who repeatedly play $2 \times 2$ coordination games with their current partners. Agents care about the difference between the sum of stage-game payoffs and network costs, which are assumed to be linearly increasing in the network size.

If the cost of forming a link is sufficiently small, net (i.e. after costs) payoffs always increase in the size of the network and agents choose to interact with as many partners as possible. Therefore, full coordination and complete networks arise. Conversely, the case of very large unit link costs is typically not explored because network externalities will be always negative and agents will prefer to hold empty networks (if they are given the option not to play the game). In both cases, no network size effects on payoffs are present and the regime of network externalities depends on the level of unit link costs.

In this paper, on the contrary, we present a dynamic, non-cooperative, model of coordination and endogenous partner selection where network externalities become eventually negative as network sizes increase. In particular, we assume that there exist (negative) network size effects on payoffs from interaction: an agent currently playing strategy $s$ who faces two networks characterized by the same number of partners choosing $s$ will earn a higher gross payoff if she chooses the smallest one. Therefore, agents could be willing to enlarge their network size if the latter is relatively small, while they will eventually prefer to shrink it (no matter strategies played) if the number of partners goes beyond some endogenously determined threshold.

This property, together with the assumption that agents cannot refuse to play the game, allows us to address the issue of coordination in presence 'locally' positive but 'globally' negative network externalities. Indeed, in many circumstances, individual net payoffs might eventually fall as the network size increases irrespective of pure strategies played by the agents either: (i) because of congestion problems entailed by the physics of the network; or (ii) because the net added value of any new player is negligible to each individual belonging to the network (see e.g. Gupta et al., 1995; for a more general perspective, cf. Economides, 1996; Arnott and Small, 1994). In particular, players may prefer to avoid unwanted interactions as unknown agents may 
entail risks (e.g. a higher likelihood of computer viruses). Moreover, whenever agents are status seeking and aspire to be similar to their peers, individuals might dislike coordination if the group to which they belong becomes too crowded (see e.g. Cowan et al., 1998).

Our approach departs from existing models of coordination with endogenous networks in a few other respects. First, in existing models no underlying socio-economic geography prevents (or restricts) interactions between 'distant' agents (cf. Jackson and Watts, 2000). On the contrary, we study a framework where agents are spatially located on the nodes of a circle and can only play $2 \times 2$ pure coordination games with their nearest neighbors.

Second, we study societies where neighborhood adjustment is endogenous but relatively not frequent. More precisely, we suppose that in each time period players are allowed either to update their current pure strategy (given the current interaction structure) or, with some exogenously fixed probability, to simultaneously adjust strategies and neighbors. Conversely, existing formalizations typically assume that the speed at which agents can change their partners is the same as the pace at which they update their strategy.

Third, we suppose that players act myopically and update pure strategies and/or neighbors by best-responding to their current environment in a completely deterministic way (i.e. without mistakes). ${ }^{1}$ As holding neighbors is assumed to be costly (total cost is proportional to neighborhood size), a player will simply adjust her current strategies and/or her current set of partners by comparing net payoffs from coordination.

Finally, we introduce a 'local' neighborhood formation process. Indeed, unlike Droste et al. (2000), we assume that agents update their neighbors' set by adding to (or discard from) it only those players who are located close to the boundaries of the neighborhood. More precisely, if an agent interacts at time $t-1$ with all individuals placed no farther than $r>1$ nodes from her, she will only consider either to shrink it to $r-1$; or enlarge it to $r+1$; or keep it unchanged (while simultaneously choosing her current strategy). Local neighborhood adjustment reflects here the well-documented inertia of many social relationships (see e.g. Miller, 1963; Akerlof, 1997). Notice also that, while in existing models players can directly choose their partners in a sequence of additions and deletions of single links, here local neighborhood adjustment implies that players can only indirectly choose whom to interact with by adjusting their 'interaction window'.

We start by studying a basic setup where agents play a purely symmetric coordination game, i.e. a coordination game where both strategies are equivalent with respect to Pareto efficiency and risk-dominance. The goal is to study whether endogenous neighborhood formation alone has a significant impact on long-run coordination levels (e.g. the relative frequency of agents playing the same strategy) and interaction patterns (e.g. low vs. large neighborhood sizes).

\footnotetext{
${ }^{1}$ This contrasts with adaptive play with mistakes studied in Goyal and Vega-Redondo (2001) and Jackson and Watts (2000), while is in line with fair parts of Droste et al. (2000) and previous work by Blume (1993) in the context of static interaction structures.
} 


\section{The model}

Consider a finite set of agents $I=\{1,2, \ldots, N\}, N>5$. Agents are spatially distributed on the nodes of a one-dimensional lattice with periodic boundaries (i.e. a circle). We assume a one-to-one relation between the nodes of the lattice and the set of agents. Hence, individual labels $i=1,2, \ldots, N$ coincide with locations on the circle. Time is discrete. At each $t=1,2, \ldots$ any agent plays a pure $2 \times 2$ coordination game against her current partners. More precisely, if agent $i$ plays $s_{i} \in\{-1,1\}$ when agent $j$ 's strategy is $s_{j} \in\{-1,1\}$, her stage-game payoff is given by

$$
G\left(s_{i}, s_{j}\right)= \begin{cases}1 & \text { if } s_{i}=s_{j} \\ 0 & \text { if } s_{i} \neq s_{j}\end{cases}
$$

Any agent $i$ is completely characterized by her set of partners $V_{i t} \subset I$ and her current strategy $s_{i t} \in S=\{-1,+1\}$. We assume that agent $i$ 's partners set consists of all players located within a symmetric neighborhood with radius $r_{i t}$. More formally:

$$
V_{i t}=V\left(r_{i t}\right)=:\left\{j \in I: 0<\min \{|j-i|, N-|j-i|\} \leqslant r_{i t}\right\},
$$

where $r_{i t} \in R(N)=\{1,2, \ldots, \tilde{r}\}$ and $\tilde{r}=\lfloor N / 2\rfloor$. Notice that, as in principle $r_{i t} \neq r_{j t}$ for any two $i \neq j$, one might have that $i \in V_{j t}$ but $j \notin V_{i t}$. Since $V_{i t}$ only depends on $r_{i t}$, we define the state of any agent $i$ at time $t$ as

$$
\left(s_{i t}, r_{i t}\right) \in S \times R(N) .
$$

The economy will be in turn characterized at any $t$ by its configuration

$$
\Omega_{t}=\left(s_{i t}, r_{i t}\right)_{i \in I},
$$

where $\Omega_{t} \in \Gamma(N)=(S \times R(N))^{N}$. As a final piece of notation, let $|\Gamma(N)|$ be the cardinality of $\Gamma(N)$.

In each time period, an agent whose state is $\left(s_{i t}, r_{i t}\right)$ earns a (net) payoff

$$
\pi_{i t}\left(s_{i t} ; r_{i t} \mid \Omega_{t}\right)=\left[\frac{1}{\left|V\left(r_{i t}\right)\right|^{\alpha}} \sum_{j \in V\left(r_{i t}\right)} G\left(s_{i t}, s_{j t}\right)\right]-\beta\left|V\left(r_{i t}\right)\right|,
$$

where $G\left(s_{i t}, s_{j t}\right)$ is defined in (1), $\left|V\left(r_{i t}\right)\right|$ is the cardinality of $V\left(r_{i t}\right)$ and $\beta>0$ is the unit cost per neighbor (i.e. the cost of keeping active at $t$ the link to any agent $j \neq i$ ).

The parameter $\alpha \in[0,1]$ governs the nature of network externalities in the model. To see why, let us consider two extreme cases. On the one hand, if $\alpha=0$, there are no neighborhood size effects on gross payoffs (i.e. the term in square brackets): if the number of agents playing $s_{i t}$ in $V\left(r^{\prime}\right)$ and $V\left(r^{\prime \prime}\right)$ is the same, then gross payoffs do not change. Therefore, if $\beta>1$ the system is characterized by negative network externalities (NNE henceforth) because net payoffs are strictly decreasing with $r_{i t}$ (and thus with the number of agents playing $s_{i t}$ in $V$ ) irrespective of strategy configuration. Conversely, if $\beta<1$ (and sufficiently small) network externalities are 'always positive': net payoffs are strictly increasing in the number of neighbors choosing $s_{i t}$.

On the other hand, if $\alpha=1$, gross payoffs from playing $\left(s_{i t}, r_{i t}\right)$ are negatively affected by the size of the neighborhood. For a given strategy configuration, $\pi_{i t}$ might indeed increase if neighborhood sizes are not too large, because gross gains from local coordination can offset costs. However, they will eventually fall, irrespective of currently 
played strategies, as neighborhood size grows. In this case the system is potentially characterized by 'locally' positive and 'globally' negative network externalities.

In this paper, we will primarily focus on the case $\alpha=1$ (i.e. very strong neighborhood size effects). The case $\alpha \in[0,1)$ will be briefly discussed in the conclusions (see however Fagiolo, 2001 for a more detailed analysis).

When $\alpha=1$, as $\left|V\left(r_{i t}\right)\right|=2 r_{i t}$ and $G\left(s_{i t}, s_{j t}\right)=1$ if and only if $s_{i t}=s_{j t}$, payoffs in (3) read

$$
\pi_{i t}\left(s_{i t} ; r_{i t} \mid \Omega_{t}\right)= \begin{cases}x_{i}\left(r_{i t}\right)-\gamma r & \text { if } s_{i t}=+1, \\ 1-x_{i}\left(r_{i t}\right)-\gamma r & \text { if } s_{i t}=-1,\end{cases}
$$

where $\gamma=2 \beta$ ('unit cost', in the following) and $x_{i}\left(r_{i t}\right) \in[0,1]$ is the share of agents in $V\left(r_{i t}\right)$ currently playing +1 ('relative frequency' in the following). Notice that NNE effects become stronger if: (i) $\gamma$ increases given $N$ and $r$; (ii) $r$ increases given $\gamma$. Since everything else being constant the attainable range of $r$ will increase with $N$, a larger population size may imply larger NNE effects as well.

Let us turn now to describe the dynamics of the model. At time $t=0$, suppose to draw an initial configuration $\Omega_{0}$ from $\Gamma(N)$ with (uniform) probability $|\Gamma(N)|^{-1}$. Assume also that, at the beginning of any period $t+1$ an agent (say $i$ ) is drawn from $I$ with (uniform) probability $N^{-1}$. We suppose that, with probability $1-\theta \in(0,1], i$ is allowed to update her current strategy $s_{i t}$ only (given $r_{i t}$ ), while with probability $\theta \in(0,1]$ she can simultaneously update both $s_{i t}$ and $r_{i t}{ }^{2}$

Consider first strategy updating only. We assume that agent $i$ best-responds (without mistakes) to the current local configuration:

$$
s_{i, t+1} \in \underset{s \in\{-1,+1\}}{\arg \max } \pi_{i t}\left(s ; r_{i t} \mid \Omega_{t}\right) .
$$

We also suppose that agents are change-averse, i.e. they stick to their current choice when ties occur.

Second, an agent drawn to update both $s_{i t}$ and $r_{i t}$ locally adjusts her neighborhood radius, together with her current strategy. More formally, we suppose that given $\left(r_{i t}, s_{i t}\right)$, agent $i$ will only have (for both $s=s_{i t}$ and $-s_{i t}$ ) the following three options: (i) shrinking to $r_{i t}-1$; (ii) keeping $r_{i t}$; or (iii) enlarging to $r_{i t}+1$. Agent $i$ 's next period state will therefore be

$$
\begin{aligned}
& \left(s_{i, t+1}, r_{i, t+1}\right) \in \quad \arg \max \quad \pi_{i t}\left(s ; r \mid \Omega_{t}\right), \\
& s \in\{-1,1\} \\
& \text { s.t. } \quad r \in R(N):\left|r-r_{i t}\right| \leqslant 1 .
\end{aligned}
$$

As happens in action updating, we suppose that players are change-averse. ${ }^{3}$

\footnotetext{
${ }^{2}$ All results presented in the next sections are not qualitatively altered if one instead assumes that, in each period $t$, an agent currently playing $\left(s_{i, t}, r_{i, t}\right)$ is allowed with probability $\theta \in(0,1)$ to revise only her current neighborhood size given the current strategy configuration (i.e. she is not allowed to change strategy and radius at the same time).

${ }^{3}$ Furthermore, we assume for the sake of simplicity that, whenever $\left(s, r_{i, t}+1\right)$ and $\left(s, r_{i, t}-1\right)$ are the unique solutions of (6) for $s \in\{-1,1\}$, agents always choose $\left(s, r_{i, t}-1\right)$. This assumption does not crucially affect the dynamic properties of the system (see Appendix A).
} 
Given any initial conditions $\Omega_{0} \in \Gamma(N)$, Eqs. (2), (4)-(6) define a Markov chain $\left\{\Omega_{t}, t \geqslant 1\right\}$ with finite state-space $\Gamma(N)$. In the following section we will start exploring its long-run behavior. We first characterize the set of steady states of the dynamic process (i.e. absorbing states of the associated Markov chain). Second, we show that this set is non-empty (but, possibly, very large) and that the system almost always converges to a steady state in finite time. Finally, we ask whether the society can be absorbed in the long-run by a SS displaying full vs. partial coordination and homogeneous vs. heterogeneous neighborhood structures.

\section{Steady states, convergence and coordination}

The best-response dynamics governing the evolution of the system exhibits a nonempty set of steady states (i.e. absorbing states for the associated Markov chain $\left\{\Omega_{t}, t \geqslant 1\right\}$ ). To see why, define a steady state (SS) of the system as a global configuration $\Omega^{*}=\left\{\left(s_{i}^{*}, r_{i}^{*}\right), i \in I\right\}$ such that any player $i \in I$ has no incentive to unilaterally move away from $\left(s_{i}^{*}, r_{i}^{*}\right)$. More formally:

Lemma 1. (Characterization of steady states). Denote by $x_{i, r_{i}^{*}}^{*}=x_{i}\left(r_{i}^{*}\right)$ local relative frequencies associated to the configuration $\Omega^{*}$. Then $\Omega^{*}$ is a SS for $\left\{\Omega_{t}, t \geqslant 1\right\}$ if and only if:

1. For any $i \in I$ s.t. $s_{i}^{*}=+1$ :

$$
\begin{array}{lr}
r_{i}^{*}=1: & x_{i, r_{i}^{*}}^{*} \geqslant \max \left\{-\gamma+x_{i, 2}^{*}, \frac{1}{2},-\gamma+1-x_{i, 2}^{*}\right\}, \\
1<r_{i}^{*}<\tilde{r}: & x_{i, r_{i}^{*}}^{*} \geqslant \max \left\{\gamma+x_{i, r_{i}^{*}-1}^{*}, \gamma+1-x_{i, r_{i}^{*}-1}^{*},\right. \\
& \left.\frac{1}{2},-\gamma+x_{i, r_{i}^{*}+1}^{*},-\gamma+1-x_{i, r_{i}^{*}+1}^{*}\right\}, \\
r_{i}^{*}=\tilde{r}: & x_{i, r_{i}^{*}}^{*} \geqslant \max \left\{\gamma+x_{i, \tilde{r}-1}^{*}, \frac{1}{2}, \gamma+1-x_{i, \tilde{r}-1}^{*}\right\} .
\end{array}
$$

2. For any $i \in I$ s.t. $s_{i}^{*}=-1$, the $l$ hs in the above inequalities are satisfied if $x_{i, r_{i}^{*}}^{*}$ is replaced by $\left(1-x_{i, r_{i}^{*}}^{*}\right)$.

Proof. Since $\theta>0$, if $\left(s_{i}^{*}, r_{i}^{*}\right)$ is stable with respect to neighborhood updating, it will be also stable for strategy updating. Hence, it suffices to impose the condition $\left(s_{i, t+1}, r_{i, t+1}\right)=\left(s_{i}^{*}, r_{i}^{*}\right)$ in Eq. (6) and solve for $x_{i}\left(r_{i}^{*}\right)$.

Consider now the set $\Gamma^{*}(\gamma, N)$ of all $\Omega^{*} \in \Gamma(N)$ satisfying SS conditions (notice that $\Gamma^{*}$ only depends on $(\gamma, N)$ because SS conditions are not affected by $\left.\theta\right)$. For any $(\gamma, N), \Gamma^{*}(\gamma, N)$ is non-empty as it always contains (at least) both configurations $\Omega^{1}=(1,1)_{i \in I}$ and $\Omega^{-1}=(-1,1)_{i \in I}$. Indeed, if all agents choose the same strategy, then $x_{i}(1) \in\{0,1\} \forall i$ and gross payoffs reach their maximum. Hence, no agent has an incentive to enlarge her current radius.

More importantly, as shown in Proposition 2, the dynamic process $\left\{\Omega_{t}, t \geqslant 1\right\}$ cannot cycle and the society will almost always converge in finite time to a SS for any $(\theta, \gamma, N)$. The reason why this happens stems from two observations. First, in each time period one and only one agent can update her current state by best-replying to local strategies profiles. Second, tie-breaking rules defined in Section 3 imply that individual best replies are unique given the current configuration. Hence, the resulting best-reply 
strategy revision process governing the evolution of configurations $\Omega$ is characterized by the 'unique best-reply property' (see Blume, 1995). For such class of Markov processes, there exists a one-to-one correspondence between absorbing states and ergodic sets (i.e. all configurations which are not SS are transient) so that finite-time convergence to a $\mathrm{SS}$ is a probability-one event.

Proposition 2 (Convergence to SS). For any given $N>5, \theta \in(0,1], \gamma>0$ and $\Omega_{0} \in \Gamma$ $(N)$, the process $\left\{\Omega_{t}, t \geqslant 1\right\}$ defined by Eqs. (2), (4)-(6) will almost always converge in finite time to an absorbing state $\Omega^{*} \in \Gamma^{*}(\gamma, N)$.

Proof. See Appendix A.

Are there any other SS apart from the two full-coordination ones? As the following example shows, for any given $(\gamma, N)$, the two configurations $\Omega^{+1}$ and $\Omega^{-1}$ are not the unique absorbing states of the process. Indeed, even for small populations, there might exist many SS characterized by partial coordination, no matter the level of unit $\operatorname{costs} \gamma$. In turn, low coordination SS may sustain, depending on $(\gamma, N)$, many different neighborhood structures.

Example 3. Suppose that $N=11$ (i.e. $\tilde{r}=5$ ). Consider the three configurations depicted below (the state of each agent $\left(s_{i}^{*}, r_{i}^{*}\right)$ is reported beside each little circle). By replacing local relative frequencies $x_{i}\left(r_{i}^{*}\right)$ in $S S$ conditions $(1)$, it is easy to see that $(A)$ and $(B)$ are both SS for all $\gamma \geqslant 0$ (cf. Lemma B.5 in the Appendix). $A$ and $B$ are both characterized by low coordination and homogeneous, minimal, interaction structures (all agents hold $\left.r_{i}^{*}=1\right)$. Population $A$ entails the lowest possible coordination level ( 6 agents playing $+1,5$ playing -1 ), while $B$ reaches higher (although partial) coordination.
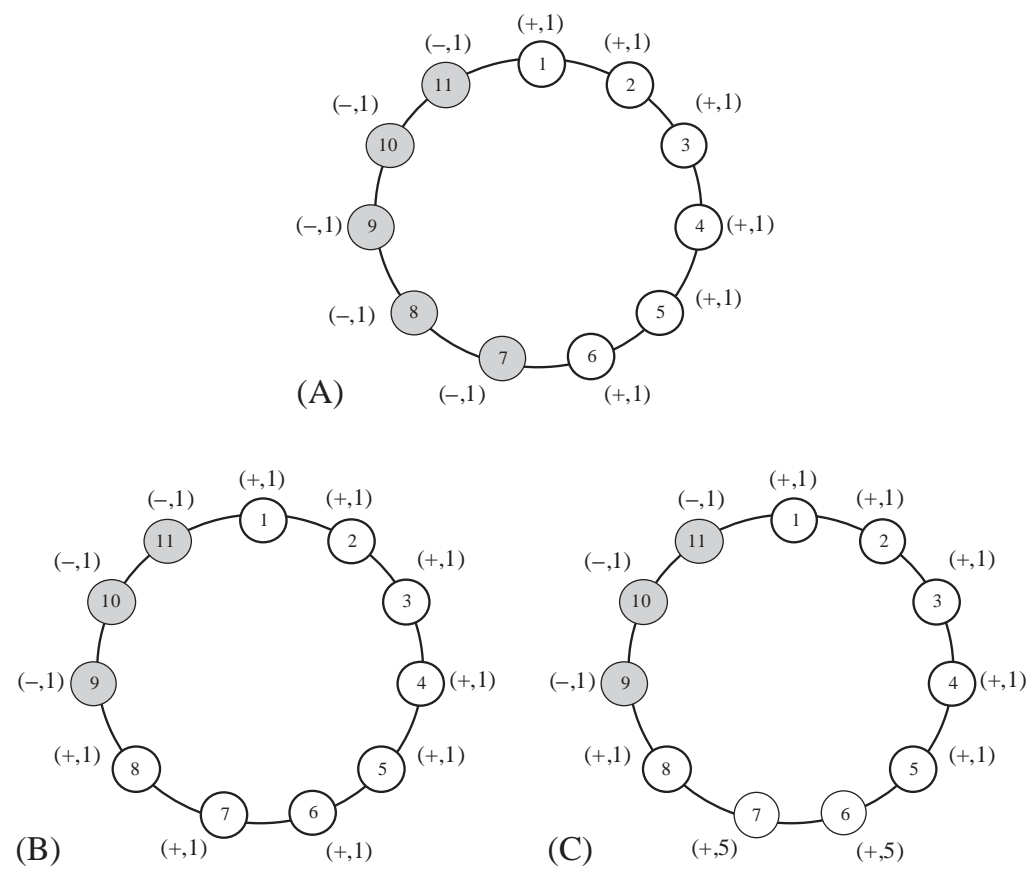
Population $C$ exhibits the same strategy configuration as B. Neighborhood structure in $C$ differs from $B$ because in $C$ agents 6 and 7 interact with everyone else $\left(r_{i}^{*}=\tilde{r}, i=6,7\right)$. This choice is a best-response for $i=6,7$ if and only if unit costs are sufficiently low (more precisely iff $\gamma \leqslant 3 / 40$ ). In this range of unit costs, both $B$ and $C$ are SS: the same low-coordination strategy configuration can sustain more than one interaction structure.

These findings contrasts with existing literature (see e.g. Goyal and Vega-Redondo, 2001; Jackson and Watts, 2000), where only full coordination (with complete networks) can arise. In our model, on the contrary, the presence of spatial constraints to neighborhood adaptation (e.g. local radius adjustments and lattice structure), coupled with negative network externality effects and agents' change-aversion, allows for the emergence of low-coordination SS (possibly with heterogeneous neighborhoods $){ }^{4}$

As the foregoing example suggests, the set $\Gamma^{*}(\gamma, N)$ might generally be very large. For instance, it is easy to see that homogeneous, minimal, interaction structures $\left(r_{i}^{*}=\right.$ $1 \forall i$ ) can support many non-minimal strategy configurations. More precisely (see Fagiolo, 2001 for a formal proof), for all $\gamma \geqslant 0$, any strategy configuration involving $N-k$ consecutive agents on the circle playing $s$ (and a cluster of $k$ agents playing $-S$ ) can be sustained as a SS by $r_{i}^{*}=1 \forall i$ for all $k>2$. Similarly, societies characterized by partial coordination are able to sustain a huge number of non-minimal (heterogeneous) interaction structures (i.e. s.t. $\exists i: r_{i}^{*}>1$ ) for sufficiently low link costs.

Since our main goal is to study aggregate levels of coordination and neighborhood sizes, we will not try here to fully characterize the set $\Gamma^{*}(\gamma, N)$ in terms of spatial patterns of coordination and interaction structures (e.g. existence, size and stability of strategy enclaves or niches, degree of overlaps between neighborhoods, etc.). Conversely, we will attempt to classify SS as to whether: (i) the associated strategy configurations display full vs. partial coordination; (ii) SS neighborhood configurations are homogeneous and minimal or not. The idea is that, thanks to symmetry of $G$ and roto-translation invariance of our lattice, one can focus on statistics of SS strategy and radius configurations (e.g. average coordination, average neighborhood size, etc.) instead of their internal fine structure, because the former are typically invariant across many SS.

In the next proposition we will therefore answer the following questions: Can full coordination arise in a SS together with interaction structures different from the homogeneous, minimal, one? Is it possible to observe in a SS partial coordination together with non-minimal neighborhood structures? And, finally: For which parameter values partial coordination configurations can support non-minimal neighborhoods?

\footnotetext{
${ }^{4}$ Notice that if players 6 and 7 of population $C$ in Example 3 could always choose any $r \in\{1, \ldots, 5\}$, they would both immediately switch to $r_{i}=5$. Hence, dropping the assumption of local radius adjustments may change the set of SS. A study of alternative neighborhood adaptation rules is contained in Fagiolo (2001).
} 
Proposition 4. Consider a SS $\Omega^{*} \in \Gamma^{*}(\gamma, N)$. Then:

1. For any $(\gamma, N)$, full coordination necessarily implies in a $S S r_{i}^{*}=1, \forall i$.

2. For any $(\gamma, N)$, partial coordination and homogeneity of neighborhood sizes can only arise in a $S S$ if $r_{i}^{*}=1 \forall i$.

3. Partial coordination can arise in a SS with non-minimal (heterogeneous) neighborhood sizes only if $(\gamma, N): \gamma(N-5)(N-3) \leqslant 1$.

Proof. See Appendix B.

Some remarks are in order. Notice first that NNE effects prevent any network different from the minimally connected one (and thus also the complete one) to sustain full coordination in a SS, no matter how small unit costs are. Moreover, heterogeneous strategy configurations cannot sustain a homogeneous neighborhood structure with $r_{i}^{*}>1, \forall i$. This is because the larger neighborhood sizes held by two adjacent agents who play opposite strategies, the larger the number of common neighbors playing +1 who are needed in order for that configuration to be locally stable with respect to strategy updating (see Fagiolo, 2001). This prevents sustainability of interaction structures with $r_{i}>1 \forall i$. Finally, heterogeneous strategy configurations can sustain heterogeneous neighborhoods only if NNE are not too strong. Since network externalities become eventually negative when neighborhood sizes increase for a given $N$, any agent holding a $r$-neighborhood will increasingly prefer not too enlarge as $\gamma$ increases given $r$ (or, equivalently, as $r$ increases given $\gamma$ ). In the extreme case (i.e. $2 \gamma(r-1)>1$, $r \geqslant 2$ ), the agent will always shrink to $r-1$ irrespective of the local strategy profile. Therefore, heterogeneous strategy configurations can sustain radii larger than one only if $\gamma$ is sufficiently small. In such situations, network externalities may be perceived to be positive even at large distances. Some agents will then have an incentive to enlarge their information 'window' up to values close to $\tilde{r}$. On the other hand, as $\gamma$ increases for given $N$, NNE tend to dominate even for small neighborhood sizes and agents always shrink their radius down to $r=1$.

Proposition 4 allows us to conclude that SS can belong to three classes. First, the system may converge to full coordination, and homogeneous, minimal, neighborhoods (i.e. $\Omega^{1}$ and $\Omega^{-1}$ ). Second, the population may end up in (one of the many) partial coordination configurations with homogeneous, minimal neighborhoods $\left(r_{i}=1 \forall i\right.$ ). Finally, if NNE are not too strong, partial coordination may occur together with non-minimal interaction structures. Notice that in the first two cases aggregate neighborhood cost is minimized $(N \gamma)$, while in the last case the SS is socially inefficient because aggregate neighborhood cost is strictly larger than $N \gamma$.

\section{The long-run behavior of the system: simulation results}

In the last section we have shown that, especially for small $\gamma$, the system can in principle exhibit very different aggregate coordination levels and interaction patterns. In what follows we study how system parameters (unit costs, frequency of neighborhood updating and population size) affect the way in which the dynamic process is able 
to select among SS. In particular, we are interested in assessing how likely a society might be trapped in SS characterized by full vs. partial coordination and how frequently low coordination can arise together with non-minimal, heterogeneous, neighborhood structures.

\subsection{Setup of Monte-carlo exercises}

To begin with, notice that, for any given choice of $(N, \gamma, \theta)$, the particular SS which the best-response strategy and neighborhood revision process will select depends on both initial conditions and the sequence of individual updatings. Since we assumed that $\Omega_{0}$ are random (uniform) draws from $\Gamma(N)$ and updating players are also randomly drawn from $I$ with uniform probabilities in each $t$, we can indirectly address equilibrium selection by estimating how the size of the basin of attraction of different classes of SS (e.g. full vs. low coordination ones) changes with $(N, \gamma, \theta)$, after having factored out random effects induced by initial conditions and updating sequence. ${ }^{5}$

In order to do that, we have performed an extensive set of Monte-carlo exercises to estimate (empirical) frequency distributions of a few interesting statistics computed on SS configurations. This exercise has been performed for a sufficiently large number of points $(N, \gamma, \theta)$ chosen on evenly spaced grids defined over $\gamma \in(0,2], \theta \in(0,1]$ and $N \in\{10, \ldots, 150\}$ (for a total of 800 parametrizations). For any $(\gamma, \theta, N)$, we have run $M=1000$ independent realizations of the process $\left\{\Omega_{t}, t \geqslant 1\right\}$. In each realization $m=1, \ldots, M$, we have employed as initial conditions a configuration $\Omega_{0, m}$ drawn from $\Gamma(N)$ with uniform probabilities (and independently across $m$ ). ${ }^{6}$ After the system has converged to some SS $\Omega_{m}^{*}=\left(s_{i, m}^{*}, r_{i, m}^{*}\right)_{i=1}^{N}$ (which happens with probability-one in each realization, see Proposition 2), we have computed SS statistics as follows.

Dropping for simplicity dependence of SS configurations on $\Omega_{0, m}$ and $(\gamma, \theta, N)$, define first average $S S$ coordination as

$$
\bar{c}_{m}=\left|\bar{s}_{m}\right|=\left|N^{-1} \sum_{i=1}^{N} s_{i, m}^{*}\right| .
$$

Notice that $\bar{c}_{m} \in[0,1], \bar{c}_{m}=1$ (full coordination) if and only if $\Omega_{m}^{*} \in\left\{\Omega^{1}, \Omega^{-1}\right\}$, while $\bar{c}_{m} \leqslant N^{-1}$ if the society is split in two (almost equal) uncoordinated populations. Relatedly, we are also interested in the relative frequency of being absorbed in a full-coordination configuration (i.e. in either $\Omega^{1}$ or $\Omega^{-1}$ ):

$$
h=\frac{1}{M} \sum_{m=1}^{M} 1_{\left\{\bar{c}_{m}=1\right\}},
$$

where $h \in[0,1]$ and $1_{\left\{\bar{c}_{m}=1\right\}}=1$ if $\bar{c}_{m}=1,0$ otherwise.

\footnotetext{
${ }^{5}$ Since individual strategy revision is completely deterministic, we cannot study directly equilibrium selection as did e.g. in Ellison (1993). Even if we introduced low-probability idiosyncratic "flips" in best-response rules, one should make additional, strongly restrictive, assumptions on neighborhood structures (e.g. homogeneity) in order for applying standard long run equilibrium selection tools.

${ }^{6}$ This MC sample size is sufficient both to attain a good precision of the estimates and to keep the probability of duplications in drawing initial configurations below $0.5 \%$, even for small values of $N$.
} 
Second, denote $S S$ average neighborhood size (i.e. average radius) by

$$
\bar{r}_{m}=N^{-1} \sum_{i=1}^{N} r_{i, m}^{*} .
$$

Of course, $\bar{r}_{m} \in[1, \tilde{r}]$ and $\bar{r}_{m}=1$ if and only if $r_{i, m}^{*}=1 \forall i .^{7}$

For any $(\gamma, \theta, N)$, our $M$ Monte-carlo realizations allow us to estimate the SS distributions of the bivariate $\left(\bar{c}_{m}, \bar{r}_{m}\right)$. In what follows, we will restrict our attention on how MC means

$$
(\hat{c}, \hat{r})=\left(M^{-1} \sum_{i=1}^{M} \bar{c}_{m}, M^{-1} \sum_{i=1}^{M} \bar{r}_{m}\right)
$$

as well as $h$, change with $(N, \gamma, \theta){ }^{8}$

In Section 4, we have shown that a homogeneous, minimal, interaction structure is able to sustain in a SS either one of the two full-coordination strategy configurations or one among many partial coordination ones. Since negative network externality effects are present across the whole parameter space, the system should be driven on average toward SS characterized by low (possibly minimal) neighborhood sizes. Therefore, if all SS above were equally probable ex ante (i.e. across different initial conditions and updating sequences), low levels of average coordination should on average emerge.

Moreover, system parameters should exert a significant effect on both aggregate coordination and neighborhood structures. For instance, stronger network externalities (higher $\gamma$ ) could favor smaller radii and thus affect coordination. A larger frequency of neighborhood updating $(\theta)$ may introduce more turbulence and influence SS selection. Finally, a larger $N$ might generate non-trivial size-effects: larger societies might indeed imply higher attainable neighborhood sizes and thus, everything else being constant, more widespread tendency toward neighborhood shrinking.

Given all that, in the next sub-sections we will ask the following questions: (i) How large is (on average) the likelihood of full-coordination $(h)$ in our society (and does it change across the parameter space)? (ii) How are SS average coordination and average neighborhood sizes affected by system parameters? And relatedly: (iii) Does the presence of endogenous neighborhood formation allow the society to reach larger coordination levels than if the interaction structure were exogenous and static (i.e. $\theta=0)$ ?

\subsection{How likely is full coordination?}

As noted above, one could expect the model to deliver quite a pessimistic prediction about the likelihood of observing a fully coordinated society in the long-run. The very

\footnotetext{
${ }^{7}$ Notice that focusing on averages $\left(\bar{c}_{m}, \bar{r}_{m}\right)$ only is not too restrictive. Indeed, it is easy to see that in a $\mathrm{SS}: \operatorname{var}\left(s_{i, m}^{*}\right)=1-\bar{c}_{m}^{2}$ and $\operatorname{var}\left(r_{i, m}^{*}\right)=-\tilde{r}+(1+\tilde{r}) \bar{r}_{m}-\bar{r}_{m}^{2}$. Hence, $\left(\bar{c}_{m}, \bar{r}_{m}\right)$ convey information also on second moments of coordination and neighborhood configurations.

${ }^{8}$ Space constraints prevent us from providing a detailed study of MC empirical distributions $\left(\bar{c}_{m}, \bar{r}_{m}\right)$, which is however available upon request from the author. Nevertheless, we argue, the following exercises are able to satisfactorily answer our main questions about long-run coordination and neighborhood sizes.
} 
Table 1

Montecarlo relative frequency $h$ of runs where full coordination arises. MC sample size $M=1000$

\begin{tabular}{llllll}
\hline \multicolumn{5}{c}{} & \multicolumn{4}{l}{ Population size $(N)$} & \\
\cline { 3 - 6 } & & 10 & 20 & 50 & 100 \\
\hline \multirow{3}{*}{$\gamma=0.1$} & $\theta=0.1$ & 0.884 & 0.913 & 0.990 & 0.998 \\
& $\theta=0.5$ & 0.878 & 0.834 & 0.908 & 0.990 \\
$\gamma=0.5$ & $\theta=1.0$ & 0.853 & 0.760 & 0.849 & 0.987 \\
& $\theta=0.1$ & & & & \\
& $\theta=0.5$ & 0.829 & 0.871 & 0.925 & 0.976 \\
$\gamma=1.0$ & $\theta=1.0$ & 0.687 & 0.650 & 0.749 & 0.876 \\
& & 0.569 & 0.534 & 0.632 & 0.812 \\
& $\theta=0.1$ & 0.846 & 0.865 & 0.929 & 0.966 \\
& $\theta=0.5$ & 0.694 & 0.648 & 0.757 & 0.894 \\
& $\theta=1.0$ & 0.571 & 0.532 & 0.599 & 0.807 \\
\hline
\end{tabular}

possibility of having many low-coordination SS associated to the minimal interaction structure would lead us to expect, in presence of negative network externalities pushing toward small neighborhood sizes, full coordination to arise across the whole parameter space only if initial configurations were sufficiently close to $\Omega^{1}$ or to $\Omega^{-1}$.

For instance, consider the likelihood that a system initialized with a minimal interaction structure $\left(r_{i, 0}=1 \forall i\right)$ and random strategy configuration reaches full coordination. Simulations show that this figure turns out to be (on average across all initial strategy configurations and parameters) around $14 \%$ but always smaller than $40 \%$. Since NNE drive the system toward interaction structures with small neighborhoods, one might expect to observe comparably low values for $h$ when one averages out the effect of randomly drawn initial interaction structures.

On the contrary, MC simulations point out that, if initial conditions $\Omega_{0, m}$ are drawn at random from $\Gamma(N)$, the likelihood of being absorbed by a full coordination, minimal interaction structures, SS is much higher than expected across the whole sampled parameter space. In Table 1, we report - as an illustrative example - values of $h$ as $(\gamma, \theta, N)$ vary in the relevant space and initial conditions are independently and uniformly drawn from $\Gamma(N)$ in any MC run $m=1, \ldots, 1000$. The likelihood of full coordination (and consequently of minimal interaction structures) is always greater than $50 \%$ for all parametrizations. More precisely, in $89.6 \%$ of all runs (i.e. across the whole sampled parameter space) full coordination arises and typically $h$ reaches values very close to one, especially when $\gamma$ and $\theta$ are small and $N$ is large.

These results imply that, starting from any given initial neighborhood structure and strategy configurations, agents are in general able to use neighborhood updating to coordinate their actions in a quite efficient way. In fact, if initial neighborhood sizes were minimal (or very small), agents would tend to stick to their initial radii due to NNE effects and only employ strategy updating. This, however, would generate, on average, low coordination. Conversely, the pressure of NNE allows neighborhood adjustment to drive the system toward high coordination outcomes. 


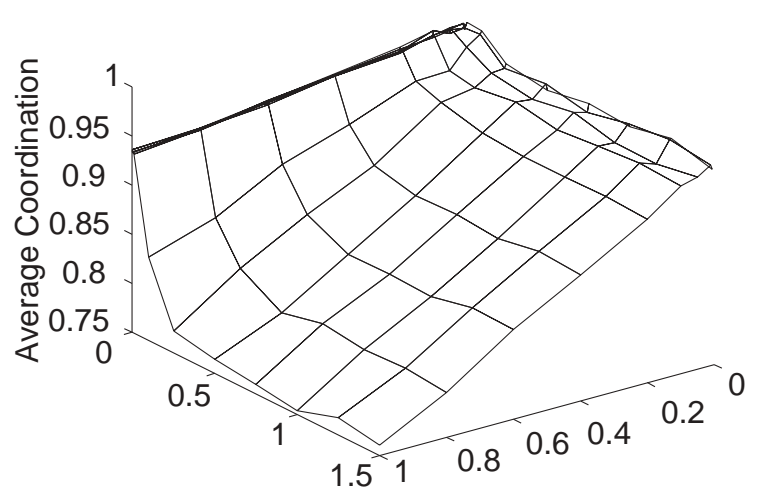

(a)

Average Coordination

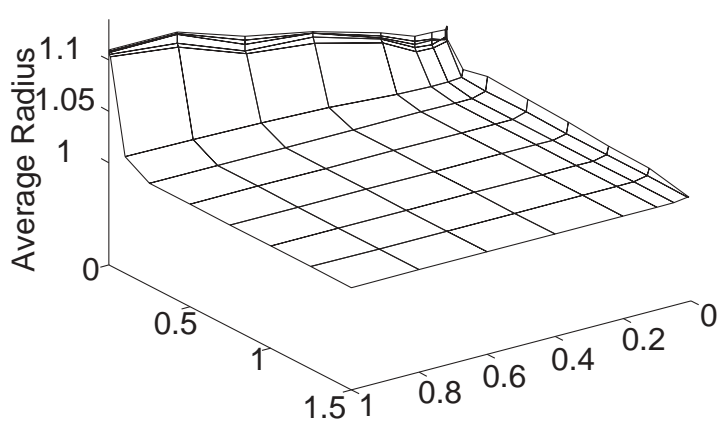

(b)

Average Radius

Fig. 1. Means of MC average coordination distributions $\left\{\bar{c}_{m}, m=1, \ldots, M\right\}$ (a) and MC average radius distributions $\left\{\bar{r}_{m}, m=1, \ldots, M\right\}$ (b) as a function of unit neighborhood cost $\gamma$ and frequency of neighborhood updating $\theta$. Population size $N=21$. MC sample size $M=1000$.

\subsection{Coordination, negative network externalities and neighborhood updating}

The foregoing evidence about the behavior of $h$ does not convey any information about actual coordination levels and neighborhood sizes (which are displayed by the system if $\Omega^{1}$ or to $\Omega^{-1}$ are not reached). We then turn now to a more detailed study of how average coordination and neighborhood $(\hat{c}, \hat{r})$ behave across the parameter space for totally random initial conditions. This exercise will also shed more light on the ways system parameters affect the patterns of coordination and interaction structures.

Let us start by exploring $(\hat{c}, \hat{r})$ as a function of unit link costs $(\gamma)$ and frequency of neighborhood updating $(\theta)$, for any given population size $(N)$. First, consistently with the above results on $h$, overall coordination levels are much higher than if we started from a minimal initial neighborhood structure. ${ }^{9}$

Second, system parameters engender a non-trivial effect on coordination and neighborhood sizes. On the one hand, larger neighborhood costs imply lower coordination and smaller neighborhood sizes. On the other hand, a smaller frequency of neighborhood updating entails higher coordination and smaller neighborhood sizes. This pattern (which arises consistently across all sampled values of $N$ ) is illustrated in Figs. 1(a) and (b), where we plot $\hat{c}$ and $\hat{r}$ against $(\gamma, \theta)$ for $N=21$.

To see why, recall that the higher $\gamma$, the stronger NNE effects faced by agents given $N$. This will induce lower neighborhood radii. Moreover, the share of agents who always tend to shrink (possibly irrespective of their local strategy configurations) increases. If agents are increasingly unable to employ neighborhood adjustment to locally coordinate, the system will tend to converge toward SS with lower values of $\hat{c}$.

The effect exerted by the frequency of neighborhood updating is even more interesting. The larger $\theta$, the higher the number of agents who always update strategy and radius together in subsequent periods, and the heavier the turbulence introduced in the

\footnotetext{
${ }^{9}$ For instance, in $84.6 \%$ of all runs (i.e. across the whole sampled parameter space) the society is absorbed in SS characterized by $\bar{c}_{m} \geqslant 0.9$, while in $99.1 \%$ of runs the scaled average radius $\bar{R}_{m}=\tilde{r}^{-1} \bar{r}_{m}$ is smaller than 0.50 . On the contrary, if we start from a minimal initial neighborhood structure, the average coordination level is only $32.4 \%$.
} 


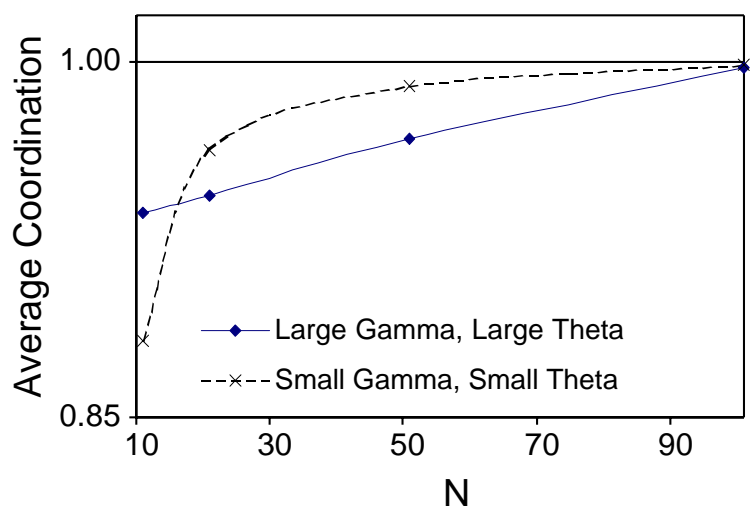

(a)

Average Coordination

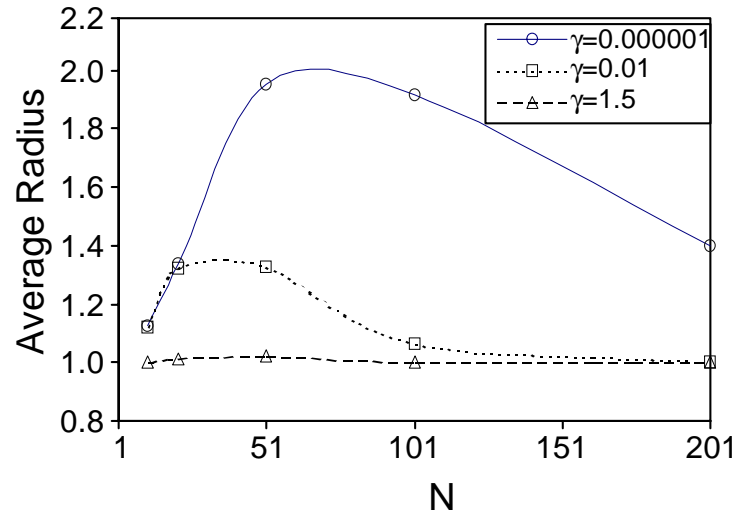

(b)

Average Radius

Fig. 2. Means of MC average coordination distributions $\left\{\bar{c}_{m}, m=1, \ldots, M\right\}$ (a) and MC average radius distributions $\left\{\bar{r}_{m}, m=1, \ldots, M\right\}$ (b) as a function of the population size $(N)$. Parameter setups: $\gamma \in\{10 E-07,1.5\}, \theta \in\{0.1,0.9\}, M=1000$.

evolution of configurations. If NNE effects are sufficiently strong, agents will tend to shrink their neighborhoods very fast. This prevents them to satisfactorily exploit the initially more connected interaction structure to better coordinate. Therefore, if $\theta$ is large, the system quickly converges toward a SS, but it may be more likely trapped in a low-coordination configuration. On the contrary, if $\theta$ is very low, agents almost always update their strategy for their given $r$. Thus, they can exploit their larger initial radii for a longer period of time and possibly achieve a high-coordination SS. From then on, rare neighborhood updating events act as a 'tremble' that de-locks the system from partial coordination states and pushes it toward full coordination.

\subsection{Coordination, interaction structures, and population size}

Let us now turn to explore the effects on $(\hat{c}, \hat{r})$ of a growing population size $N$ for given $(\gamma, \theta)$. As noted above, a larger population size implies, everything else being constant, stronger NNE effects. Simple computations show that any agent holding neighborhoods $r>1+(2 \gamma)^{-1}, r \geqslant 2$, will always shrink to $r-1$, irrespective of local relative frequencies and independent of $N$. Thus, as $N$ grows, the range of attainable radii increases (as $\tilde{r}=\lfloor N / 2\rfloor$ ) and the inequality above is increasingly satisfied for any given $\gamma$.

Consequently, a trade-off arises. Larger $N$ should imply (due to a size effect) larger neighborhood sizes. However, NNE effects become more pervasive as $N$ increases, thus leading to an increasing likelihood of SS characterized by smaller neighborhood sizes. As Fig. 2(b) illustrates, MC exercises confirm that this conjecture consistently holds in all parametrizations. Notice that, given any $(\gamma, \theta), \hat{r}$ first increases and then converges toward 1 in all setups. However, this pattern emerges more clearly the smaller is $\gamma$ : the weaker NNE effects, the more the size-effect will dominate.

Similarly, a larger $N$ should induce weaker average coordination levels through increasing NNE effects. On the contrary, MC exercises show that average coordination 
is non-decreasing in $N$ and tends to values close to 1 , for all sampled values of $(\gamma, \theta)$ : see for an example Fig. 2(a).

Once again, a trade-off seems to arise in our model. On the one hand, larger societies are characterized by a higher share of agents who do not employ neighborhood updating and thus find increasingly harder to coordinate among them. On the other hand, the higher current average neighborhood sizes, the lower the likelihood to sustain in a SS a partial coordination configuration. ${ }^{10}$ Thus, as $N$ grows in our model, partial coordination configurations tend to be less sustainable in a SS because the network of links channelling information among players is initially more connected. As illustrated by Fig. 2(a), the second effect dominates the first one, across all sampled $(\gamma, \theta)$ setups. The possibility of exploiting initial neighborhood structures characterized by average neighborhood size increasing in $N$ speeds up individual learning and favors more coordinated configurations in the long-run.

\subsection{Static vs. evolving interaction structures}

In our model, the society is able to effectively coordinate despite the presence of negative network externalities. But how important is the contribution of endogenous neighborhood formation? To answer this question, we compare here average coordination levels attained by populations where neighborhood updating is allowed (denoted by $\hat{c}_{\theta>0}$ ), with those reached by systems where interaction structures are static (i.e. $\hat{c}_{\theta=0}$ ). Recall that computer simulations show that coordination is typically decreasing in $\theta$. Thus, one might expect endogenous neighborhood formation to have a negative impact on overall coordination.

To begin with, we follow Ellison (1993) and consider the benchmark case of a homogeneous initial interaction structures (i.e. $r_{i 0}=r, r \geqslant 1$ ). If $\theta=0$, agents will only adjust their strategy using our deterministic best-response rule. On the contrary, when $\theta>0$, agents will adjust both strategy and radius, given a randomly drawn initial strategy configuration but the same initial interaction structure $\left(r_{i 0}=r, r \geqslant 1\right)$.

Consider first what happens if the population size is kept constant. If the interaction structure is static and $r$ tends to $\tilde{r}$, the communication network tends to the complete one and thus $\hat{c}_{\theta=0}$ increases toward one. However, as illustrated in Fig. 3(a) for the case $N=11$, average coordination in a system with neighborhood adjustment also increases with $r$. More importantly, $\hat{c}_{\theta>0}>\hat{c}_{\theta=0}$ for all $r<\tilde{r}$. This result consistently arises in all sampled population sizes. Notice also that Fig. 3(a) plots $\hat{c}_{\theta>0}$ in its worst-case scenario $(\theta=1)$ : since $\hat{c}$ is decreasing in $\theta$ and $\hat{c}_{\theta=0}$ does not depend on $\gamma$, all $\hat{c}_{\theta>0}$ curves (for any given $N$ ) will lie above $\hat{c}_{\theta=0}$.

Second, suppose to increase the population size for any given $(\gamma, \theta)$. Given any initial $r$, the scaled radius $\tilde{r}^{-1} r$ will tend to zero as $N$ increases. Thus, a society facing a fixed interaction structure will experience decreasing coordination levels if it becomes

\footnotetext{
${ }^{10}$ More formally, for any pair of adjacent agents playing opposite strategies, the number $k$ of common neighbors playing +1 necessary for local stability increases with their average neighborhood size. Therefore, as $N$ grows, $k$ must grow for any pair of adjacent agents playing opposite strategies. This will systematically destroy heterogeneous strategy configurations. This result is quite general and holds even if neighborhood updating is shut down (see Fagiolo, 2001).
} 

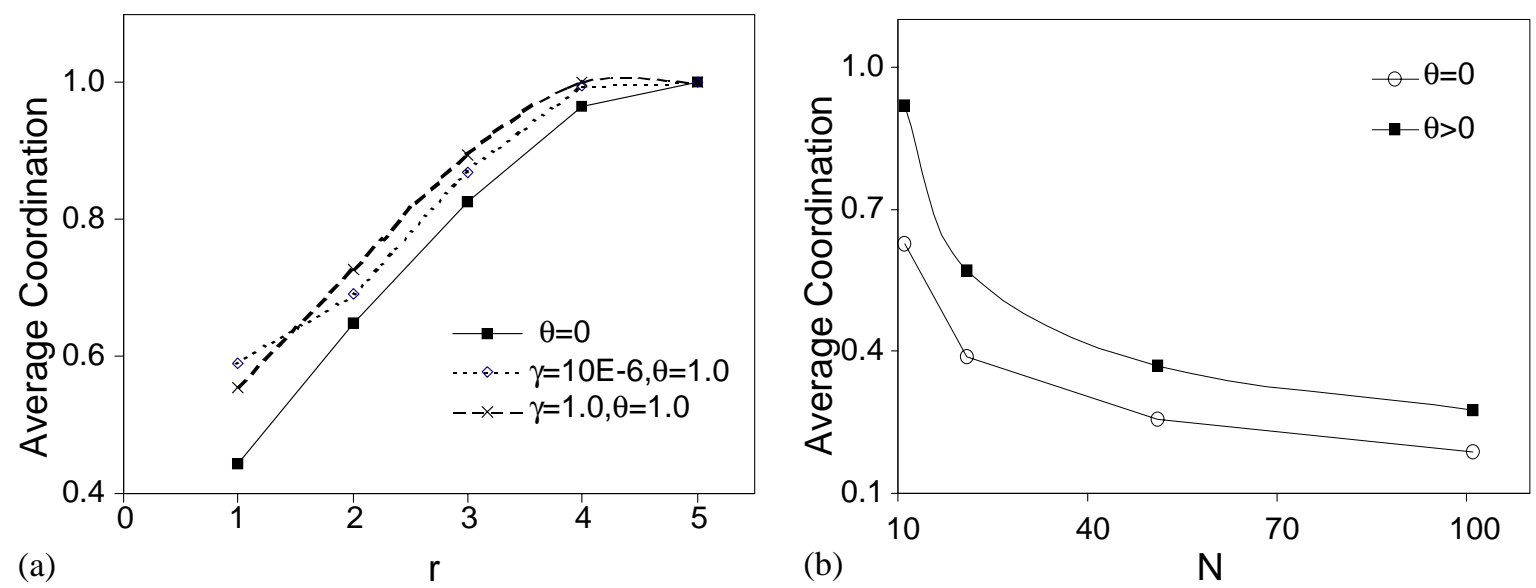

Fig. 3. Static vs. endogenous neighborhood structures with homogeneous initial neighborhood radius $r$. Panel (a): means of MC distributions of average coordination $\bar{c}_{m}$ in the case $\theta=0$ vs. $\theta>0$. Solid and dashed lines represent average coordination $\hat{c}$ as the homogeneous neighborhood radius $r$ increases (parameter setup: $N=11, M=10$ 000). Panel (b): means of MC distributions of average coordination $\bar{c}_{m}$ when $\theta=0$ vs. $\theta>0$ as $N$ changes (given $r$ ). Parameter setup: $r_{i, 0}=r=3 \forall i, M=10000$. Data for $\theta>0$ refer to $\theta=0.1$ and $\gamma=0.001$.

larger and larger because its relative connectivity falls. Since now the average initial radius does not increase with $N$, a society with endogenous neighborhood formation cannot enjoy increasing average coordination as $N$ grows. Therefore, we expect $\hat{c}_{\theta>0}$ to be decreasing with $N$ for any given $(\gamma, \theta)$. This is confirmed by MC simulations (cf. Fig. 3(b)). However, average coordination in presence of endogenous neighborhood updating always ends up to be larger than $\hat{c}_{\theta=0}$ for all sampled parameter setups (and for all initial $r$ such that $\tilde{r}^{-1} r \rightarrow 0$ as $N$ increases). Once again, the combined effects of endogenous neighborhood formation and negative local network externalities sustain better long-run coordination patterns through the exploitation of favorable local improvements.

Finally, notice that similar results can be obtained if we assumed heterogeneous initial interaction structures $\left\{r_{i 0}, i \in I\right\}$ (i.e. where $\exists i \in I$ and $j \in I, j \neq i$ such that $r_{i 0} \neq r_{j 0}$ ) for $\theta \geqslant 0$. Therefore, if players can adapt to their local environment by both choosing strategies and partners, the society attains more coordination than if interaction structures were static.

\section{Conclusions}

The details of the process governing the coevolution of expectations formation, individual choices and interaction structure might strongly affect the long-run outcome of a coordination game repeatedly played in large populations.

To investigate this issue, we have presented a model in which myopic, spatially located individuals play a pure coordination game with their nearest neighbors. We analyze a system characterized by local network externalities which might be positive when the neighborhood size is relatively small, but they eventually become negative when the latter becomes too large. We suppose that agents are spatially located and 
that, from time to time, they can adjust both current stage-game strategies and their neighborhood size.

We have shown that the system almost always converges to a set of absorbing states in which both full and partial coordination are possible. Nevertheless, as computer simulations indicate, the system is able to reach very large average coordination levels despite the presence of negative network externality effects. Furthermore, endogenous neighborhood formation allows the system to reach higher coordination than if interaction structures were exogenous. In addition, larger societies can attain higher coordination provided that they are sufficiently connected at the beginning.

The robustness of the foregoing results may depend on a number of simplifying assumptions (cf. Fagiolo, 2001 for a more detailed discussion). For instance, is the behavior of the system affected by the particular topology of interactions? In the model we assumed for the sake of simplicity that agents are located on the nodes of a one-dimensional lattice. Placing agents on lattices with dimension $v \geqslant 2$ (and assuming Von-Neumann neighborhoods) has two consequences. First, NNE become comparatively stronger than in the case $v=1$ because gross payoffs are still bounded from above by 1 while neighborhood sizes grow as $r^{v}$. Second, the number of strategy configurations which are locally sustainable when all agents hold a radius $r=1$ increases (cf. Blume, 1993). Hence, minimal interaction structures with lower coordination levels are more likely and average coordination tends to decrease with $v$. However, average coordination is still increasing with $\gamma$ and $\theta$ (for any given $N$ ), while average radius is still decreasing in $\gamma$. On the contrary, a larger population size implies lower coordination if $v \geqslant 2$ because the constraints imposed by the geography of interactions on stable strategy configurations are weaker: low coordination configurations are therefore still reachable even when $N$ grows.

Similarly, one might experiment with different neighborhood definitions. For instance, in one dimensional lattices one may allow agents to choose asymmetric neighborhoods (i.e. with different left and right radii). In this situation, agents belonging to coordinated clusters will prefer to avoid interactions with agents outside the cluster. Therefore, disconnected networks and segregation into distinct enclaves (and consequently lower coordination) might arise. More generally, when $v \geqslant 2$, one could employ different metrics in order to define neighborhoods. Simulation exercises show that this may have non-trivial consequences on aggregate coordination. For example, using hypercubic-shaped (Moore) neighborhoods instead of hyperspheric-shaped (Von Neumann) ones, generally entails much higher coordination levels.

A second important simplification concerns network externality regimes. In the paper, we have only analyzed the extreme case where $\alpha=1$. But what happens when NNE are weaker, i.e. $\alpha \in[0,1)$ ? Interestingly enough, average coordination does not dramatically decrease with $\alpha$ (for any given parameter point). Thus, positive network externalities do not allow the society to coordinate better. Moreover, for sufficiently small values of $\gamma$, the complete network becomes the unique stable interaction structure as soon as $\alpha<1$. Thus, populations characterized by $\alpha \in[0,1)$ will achieve high coordination but lower net payoffs. Conversely, strong NNE effects $(\alpha=1)$ allow for comparatively high levels of coordination together with very small neighborhood sizes, and thus higher average net payoffs. 
As a third robustness check, one might introduce individual decision rules different from deterministic best reply (e.g. best-reply with mistakes, log-linear decision rules, etc.). Along the same lines, the consequences of assuming alternative tie-breaking rules and updating schemes might be thoroughly analyzed. For example, one could introduce agents who always randomize when ties occur.

Finally, one might depart from pure-coordination settings and study how endogenous neighborhood formation influences the trade-off between Pareto-efficiency and risk-dominance in symmetric coordination games. Existing literature indicates that the restrictions exogenously imposed on the interaction structure (and on its evolution) might crucially affect long-run outcomes. On the one hand, if one assumes direct partner selection without exogenous restrictions, risk-dominant equilibria are more likely (cf. Jackson and Watts, 2000). On the other hand, if interaction structure are static but players could move among fixed locations (and thus indirectly choose their partners), Pareto efficiency is restored (see e.g. Goyal and Vega-Redondo, 2001, p. 8). Notice that the nature of endogenous neighborhood formation assumed here lies somewhere in between the latter two extreme cases. Nevertheless, as our computer simulations show, the system displays a clear tendency toward the risk-dominant equilibrium across all parametrizations. In contrast with existing results, negative network externalities are able to restore convergence to the RD outcome even when unit costs are large enough.

\section{Acknowledgements}

Thanks to Giovanni Dosi, Sanjeev Goyal, Alan Kirman, Karl Schlag, Nick Vriend, two anonymous referees, and a few participants to 'The Society for Computational Economics' Conference (Aix-en-Provence, France, 26-29 June 2002) for stimulating and helpful comments.

\section{Appendix A. Proof of Proposition 2}

Consider (wlog) individual updating rules when $\theta=1$. Given the current strategy profile, the agent drawn to update her current state $(s, r)$ faces the following relevant payoff matrix:

\begin{tabular}{llll}
\hline & $r-1$ & $r$ & $r+1$ \\
\hline$s=+1$ & $x_{i, r-1}-\gamma(r-1)$ & $x_{i, r}-\gamma r$ & $x_{i, r+1}-\gamma(r+1)$ \\
$s=-1$ & $1-x_{i, r-1}-\gamma(r-1)$ & $1-x_{i, r}-\gamma r$ & $1-x_{i, r+1}-\gamma(r+1)$ \\
\hline
\end{tabular}

If given local frequency profile $\left(x_{i, r-1}, x_{i, r}, x_{i, r+1}\right)$ no ties occur, then best-replies are unique. If, on the contrary, some ties occur, redefine the payoff matrix by adding some $\varepsilon>0$ to the entry associated to the choice preferred under the relevant tie-breaking rule (see Section 3). Once again, the resulting embedded game is such that, for any given strategy configuration, there is a unique best-response $\left(s^{*}, r^{*}\right)$ for any individual facing updating. Therefore, for any given $N>5, \theta \in(0,1], \gamma>0$ and $\Omega_{0} \in \Gamma(N)$, the Markov 
chain governing the evolution of the process $\left\{\Omega_{t}, t \geqslant 1\right\}$ satisfies the so-called 'unique best-reply property' (see Blume, 1995). For such Markov processes, we know that there is a one-to-one correspondence between absorbing states and ergodic sets. Hence, finite-time convergence to an absorbing state is a probability one event. Finally, notice that the tie-breaking assumption concerning the case when $\left(s, r_{i, t}+1\right)$ and $\left(s, r_{i, t}-1\right)$ are the unique solutions of (6) for $s \in\{-1,1\}$ (i.e. always choose $\left(s, r_{i, t}-1\right)$ ), is not very restrictive. Indeed, let $h=0,1, \ldots, 2(r-1)$ the number of agents playing +1 in $V_{r-1}$ and $k_{r}=0,1,2$ the number of agents playing +1 in the set $V_{i, r} \backslash V_{i, r-1}$. It is easy to show that a tie occurs (when $s=+1$ ), i.e.

$$
x_{r-1}-\gamma(r-1)=x_{r+1}-\gamma(r+1)>x_{r}-\gamma r
$$

iff $h \geqslant r-k_{r}$ and

$$
\gamma=\frac{(r-1)\left(k_{r}+k_{r+1}\right)-2 h}{4(r+1)(r-1)}>\frac{(r-1) k_{r}-h}{2 r(r-1)} .
$$

Numerical experiments show that the cardinality of the set of all $\gamma$ satisfying (A.1) grows as $N^{2}$. Hence, as long as $\gamma$ lies in a dense subset of $R_{+}$, the considered tie-breaking rules are not relevant and the embedded game has the unique best-reply property anyway.

\section{Appendix B. Proof of Proposition 4}

Point 1. We need to show that: (i) the configuration $\left\{\left(s_{i}, r_{i}\right)=(+1,1)\right.$, all $\left.i \in I\right\}$ is a $\mathrm{SS}$; and that (ii) given that all agents play $s_{i}=+1$ only $r_{i}=1$ all $i$ can be a SS. Given payoff symmetry we can avoid treating the case $s_{i}=-1$, all $i$. Moreover, as SS conditions for strategy updating are implied by SS conditions for neighborhood updating, it suffices to check the latter. When all $s_{i}=+1$, then $x_{i}(r)=1 \forall r=1, \ldots, \tilde{r}$. Hence, for $r=2, \ldots, \tilde{r}-1$, generic payoff matrices read

\begin{tabular}{llll}
\hline & $r-1$ & $r$ & $r+1$ \\
\hline$s=+1$ & $1-\gamma(r-1)$ & $1-\gamma r$ & $1-\gamma(r+1)$ \\
$s=-1$ & $-\gamma(r-1)$ & $-\gamma r$ & $-\gamma(r+1)$ \\
\hline
\end{tabular}

As $1-\gamma r \geqslant-\gamma r$ for any $r$ the strategy configuration $s_{i}=+1$, all $i$, is stable for any $r$. Moreover, as payoffs are decreasing in $r$, any agent will shrink until $r_{i}=1$, all $i$.

Point 2. We need to show that for any $\left\{s_{i}, i \in I\right\}$ s.t. $|\hat{s}| \neq 1$, homogeneous radius configurations are only possible if all agents choose $r_{i}=r=1$. First, notice that $r_{i}=\tilde{r}$, all $i$ cannot be a SS. If it were, then it must be that for all $i, x_{i}(\tilde{r}) \geqslant \frac{1}{2}$ if $s_{i}^{*}=+1$, while $x_{i}(\tilde{r}) \leqslant \frac{1}{2}$ if $s_{i}^{*}=-1$. Letting $x^{*}$ be the relative frequency of agents playing +1 in $I$, then $x_{i}(\tilde{r})=\left(N x^{*}-1\right) /(N-1)$ if $s_{i}^{*}=+1$ and $x_{i}(\tilde{r})=N x^{*} /(N-1)$ if $s_{i}^{*}=-1$. Hence, in a SS: $x^{*} \geqslant(N+1) / 2 N$ if $s_{i}^{*}=+1$ while $x^{*} \leqslant(N-1) / 2 N$ if $s_{i}^{*}=-1$. By heterogeneity, the two inequalities should be satisfied simultaneously, which gives a contradiction. 
Second, any configuration in which $r_{i}=2, \ldots, \tilde{r}-1$, all $i$, cannot be a SS either. If it were, SS conditions would jointly imply (cf. Lemma 1) that $x_{i}^{*}(r)>\frac{1}{2}$ if $s_{i}^{*}=+1$ and $x_{i}^{*}(r)<\frac{1}{2}$ if $s_{i}^{*}=-1$. However, if a heterogeneous configuration is to be stable, any two adjacent agents, say $i$ and $i+1$, such that $s_{i}^{*}=+1$ and $s_{i+1}^{*}=-1$ must be locally stable. As both holds a $r$-radius neighborhood, then $V_{i, r}=\{i-r, i-r+1, \ldots, i-1, i+1$, $i+2, \ldots, i+r\}$ and $V_{i+1, r}=\{i-r+1, \ldots, i-1, i, i+2, \ldots, i+r, i+r+1\}$. Hence $V_{i, r} \cap V_{i+1, r}=\{i-r+1, \ldots, i-1, i+2, \ldots, i+r\}$. Let us call $x_{c}$ the relative frequency of agents playing +1 in $V_{i, r} \cap V_{i+1, r}$ whose size is $2(r-1)$. Then, since $s_{i}^{*}=+1$ and $s_{i+1}^{*}=-1$ :

$$
x_{i}=\frac{2(r-1) x_{c}+1_{\left(s_{i-r}=+1\right)}}{2 r} \quad \text { and } \quad x_{i+1}=\frac{2(r-1) x_{c}+1+1_{\left(s_{i+r+1}=+1\right)}}{2 r},
$$

where $1_{\left(s_{j}=+1\right)}=1$ if $s_{j}=+1$ and 0 otherwise. By the inequality above, we must have that:

$$
\begin{aligned}
& 2(r-1) x_{c}+1_{\left(s_{i-r}=+1\right)}>r \\
& 2(r-1) x_{c}+1+1_{\left(s_{i+r+1}=+1\right)}<r
\end{aligned}
$$

or $r-1_{\left(s_{i-r=}=1\right)}<2(r-1) x_{c}<r-1-1_{\left(s_{i+r+1}=+1\right)}$, which is impossible as $\max \left\{1_{\left(s_{i-r}=+1\right)}-\right.$ $\left.1_{\left(s_{i+r+1}=+1\right)}\right\}=1$.

Point 3. To show point 3, we will first provide in the following lemma sufficient conditions for local SS stability. The lemma is based on the observation that in order for each individual state $\left(s_{i}, r_{i}\right)$ to be stable, only the number of agents playing +1 in $V_{i}(r-1)$, as well as the 4 agents playing +1 in $V_{i}(r) \cap V_{i}(r-1)$ and in $V_{i}(r+1) \cap V_{i}(r)$, matter.

Lemma B.5. (Characterization of local SS conditions). Assume (wlog) that $s_{i}=+1$ (symmetric results hold for $s=-1$ ). Let $V_{r} / V_{r-1}=\left\{j \in I: j \in V_{r}, j \notin V_{r-1}\right\}$. Then:

1. Suppose that $r_{i}=1$. Let $h=0,1,2$ the number of agents $i$ playing +1 in $V_{2}$ and $k=0,1,2$ the number of agents $i$ playing +1 in $V_{2} / V_{1}$. Then $r=1$ is locally stable for agent $i$ if and only if $h \neq 0$ and $\gamma \geqslant \frac{1}{4}$ if either: (i) $h=1$ and $k=0$; or (ii) $h=1$ and $k=2$.

2. Suppose that $r_{i}=\tilde{r}$. Let $h=0,1, \ldots, 2(\tilde{r}-1)$ the number of agents $i$ playing +1 in $V_{\tilde{r}-1}$ and $k=0,1,2$ the number of agents $i$ playing +1 in $V_{\tilde{r}} / V_{\tilde{r}-1}$. Then $r=\tilde{r}$ is locally stable for agent $i$ if and only if (supposing wlog $N$ odd): $k=2$, $(N-3) / 2=r-1 \leqslant h \leqslant 2 \tilde{r}-3=N-4$ and

$$
\gamma \leqslant \frac{2(N-3-h)}{(N-1)(N-3)}
$$


3. Suppose that $r_{i}=2, \ldots, \tilde{r}-1$. Let $h$ the number of agents playing +1 in $V_{r-1}$ and $k_{r}=0,1,2$ be the number of agents playing +1 in the set $V_{i, r} \backslash V_{i, r-1}$. Then $r=r_{i}$ is locally stable for agent $i$ if and only if $k_{r} \neq\{0,1\}$ and

\begin{tabular}{lll}
\hline & $k_{r+1}=0,1$ & $k_{r+1}=2$ \\
\hline$k_{r}=2$ & $h=r-1, r-2, \ldots, 2 r-3$ & $h=r-1, r-2, \ldots, 2 r-3$ \\
& $\gamma \leqslant \frac{2(r-1)-h}{2 r(r-1)}$ & $\frac{2(r-1)-h}{2 r(r+1)} \leqslant \gamma \leqslant \frac{2(r-1)-h}{2 r(r-1)}$ \\
\hline
\end{tabular}

Proof. We prove here only the case $r_{i}=1$ (proofs of cases 2 and 3 are similar but require heavier computations). Consider first SS inequalities that $(h, k)$ must satisfy in order for $(+1,1)$ to be stable for given the strategy choice $s_{i}=+1$. Since $x_{1} \geqslant \max \left\{\frac{1}{2}, x_{2}-\gamma\right\}$, then $h / 2 \geqslant \max \left\{\frac{1}{2},(h+k) / 4-\gamma\right\}$. This implies $h>1$ and $k-$ $h \leqslant 4 \gamma$, which is always satisfied for any $\gamma>0$ unless when $h=1$ and $k=2$, where we need $\gamma \geqslant \frac{1}{2}$. If we now add constraints implied by the inequalities concerning a strategy switch, then the only additional restrictions come from the fact that $x_{2} \geqslant 1-x_{1}-\gamma$, which implies $4-3 h-k \leqslant 4 \gamma$. As $h=1,2$ and $k=0,1,2$, the (lhs) of the last inequality is always $\leqslant 0$ unless $(h, k)=(1,0)$, where we need $\gamma \geqslant \frac{1}{4}$.

Combining inequalities (B.1) and (B.2) in Lemma B.5 it is easy to see that a non-minimal (heterogeneous) neighborhood configuration can be stable only if $\gamma(N-$ $3)(N-5) \leqslant 2$. By applying local SS conditions to every agent, one can show that, e.g. the following partial coordination, non-minimal neighborhoods, configuration is globally stable $(N=21)$ if $\gamma \leqslant 8 /(7 * 21):\{-1,-1,-1,+1,+1,+1,+1,+4,+4,-10,+2,+1$, $+1,-4,-1,-1,-1,-1,-1,-1,-1\}$, where $(\operatorname{sign}) x$ means $s=(\operatorname{sign}) 1$ and $r=x$.

\section{References}

Akerlof, G., 1997. Social distance and social decisions. Econometrica 65, 1005-1027.

Arnott, R., Small, K., 1994. The economics of traffic congestion. American Scientist 82, 446-455.

Blume, L., 1993. The statistical mechanics of strategic interaction. Games and Economic Behavior 5, $387-424$.

Blume, L., 1995. The statistical mechanics of best-response strategy revision. Games and Economic Behavior $11,111-145$.

Cowan, R., Cowan, W., Swann, P., 1998. Waves in consumption with interdependence among consumers. In: Schweitzer, F., Silverberg, G. (Eds.), Evolution and Self-Organization in Economics. Duncker and Humblot, Berlin.

Droste, E., Gilles, R., Johnson, C., 2000. Evolution of conventions in endogenous social networks. Working paper.

Economides, N., 1996. The economics of networks. International Journal of Industrial Organization 14 (2).

Ellison, G., 1993. Learning, local interaction and coordination. Econometrica 61 (5), 1047-1071.

Fagiolo, G., 2001. Coordination, local interactions, and endogenous neighborhood formation. LEM Working paper, 2001/15.

Goyal, S., Vega-Redondo, F., 2001. Learning, network formation and coordination. Econometric Society World Congress, 2000. 
Gupta, A., Stahl, D., Whinston, A., 1995. The internet: a future tradegy of the commons? Technical Report, The University of Texas at Austin.

Jackson, M., Watts, A., 2000. On the formation of interaction networks in social coordination games. Technical Report, Division of Humanities and Social Sciences, Caltech, Pasadena, CA and Vanderbilt University, Nashville, TN.

Kandori, M., Mailath, G., Rob, R., 1993. Learning, mutation and long run equilibria in games. Econometrica 61 (1), 29-56.

Kirman, A., 1997. The economy as an evolving network. Journal of Evolutionary Economics 7, 339-353.

Miller, D., 1963. The study of social relationships: situations, identity and social interactions. In: Kock, S. (Ed.), Psichology: A Study of a Science, Vol. 5. McGraw Hill, New York.

Skyrms, B., Pemantle, R., 2000. A dynamic model of social network formation. Proceedings of the National Academy of Sciences 97, 9340-9346.

Young, H., 1996. The economics of convention. Journal of Economics Perspectives 10 (2), 105-122.

Young, H., 1998. Individual Strategy and Social Structure. Princeton University Press, Princeton, NJ. 